\title{
Incomplete Device Returned
}

National Cancer Institute

\section{Source}

National Cancer Institute. Incomplete Device Returned. NCI Thesaurus. Code C139462.

The device was returned incompletely, lacking parts, components or accessories that would be required for appropriate testing and analysis of root causes. 